\title{
Optimal consumption and the timing of the resolution of uncertainty
}

\author{
Louis Eeckhoudt \\ University of Mons and Lille \\ Christian Gollier \\ LEERNA-INRA, IDEI, University of Toulouse \\ Nicolas Treich ${ }^{1}$ \\ LEERNA-INRA, University of Toulouse
}

May 15, 2003

${ }^{1}$ Corresponding Author. Email: ntreich@toulouse.inra.fr. Tel: +33 5611285 14. Fax: +33 5611285 20. Address: 21 all. de Brienne, Manufacture des Tabacs, 31042 Toulouse, France. 


\begin{abstract}
As is well-known, consumers want to accumulate precautionary savings in the face of income risks when their marginal utility is convex (prudence). In this paper, we explore the effect of the timing of the resolution of income uncertainty on savings. An agent faces uncertainty about his income at date $t+2$. What is the effect of being informed that the uncertainty will be resolved at date $t+1$ on the consumption at date $t$ ? We show that the effect is positive if and only if marginal utility is convex (prudence), when either the risk free rate is equal to the rate of pure preference for the present, or when the utility function is HARA. The intuition is that an early resolution of uncertainty allows for time-diversifying the risk. It therefore plays a role similar to a reduction of the income risk, whose effect on savings is negative under prudence.
\end{abstract}

JEL Classification: D81, D91 E21

Key Words: Precautionary Savings, Prudence, Uncertainty, Life-cycle. 


\section{Introduction}

Households accumulate precautionary savings in the face of future income risks. The existing literature has focused the analysis mostly on the relationship between the optimal level of current savings and the size of the future income risk. In the real world, risks borne by consumers in their life cycle are also characterized by the timing of their resolution. In this paper, we consider the standard time-additive expected utility model to examine whether consumers should save more when the resolution of uncertainty occurs later (or earlier) in their lifetime. For example, it is often the case that one doesn't know the amount that one will inherit from one's parents after their death. An interesting problem is therefore to determine the effect of a longer lifetime of the parents on the optimal consumption of their children and, more generally, on the wealth accumulation process in the economy.

On a more technical ground, the recent trend in the life cycle consumption theory that consists in disentangling permanent and transitory shocks in the household's income process provides other examples where our analysis can be useful. This trend is illustrated for example by Campbell and Mankiw (1990) and Carroll (1997). When shocks are serially correlated through time, observing the early realizations of labour incomes may provide information

about future incomes. Some young people may face a large uncertainty about the productivity of their labour before entering the market, but this uncertainty may quickly be washed away by the mere observation of their 
actual productivity early in their career. For others, the resolution of the uncertainty may be slower because their labour productivity may be less correlated through time. Our results enlighten the relationship between the speed at which this uncertainty is resolved through time and the optimal initial consumption.

The introduction of uncertainty into the classical consumption life cycle model has generated thousands of papers over the last three decades. We know since Leland (1968) and Drèze and Modigliani (1972) that an increase in future income risks à la Rothschild-Stiglitz (1970) reduces initial consumption if the marginal utility of consumption is convex. Kimball (1990) coined the term "prudence" for this assumption. It is widely recognized as a sensible assumption on consumers' preferences. Because these models have two dates, they cannot address questions related to the timing of the resolution of uncertainty. Skinner (1988), Deaton (1992) and Carroll (1994, 1997) provide clear expositions of the multi-period theory of life-cycle consumption under uncertainty. Yet they do not investigate either the question of the temporal resolution of uncertainty. The only exception is a recent paper by Blundell and Stoker (1999). Using a three-date framework, they provide approximate solutions for optimal consumption choices under constant relative risk aversion. Their simulations show that there is more precautionary savings when the uncertainty is in the middle period than when the uncertainty is in the distant period. Observe that moving the risk from one period to another has two different effects. There is first an income effect, since moving incomes 
over time affects their net present value. The second effect comes from the change in the timing of the resolution of the uncertainty. In this paper, we focus only on this second effect.

The punchline of this paper is to show that consumers should save less when an earlier resolution of uncertainty is anticipated. There is a simple intuition for why an early resolution of uncertainty should reduce precautionary savings. This intuition relies on the notion of "time-diversification", or "selfinsurance". Risks that are realized earlier can be disseminated over more periods. Namely, a risk $\widetilde{x}$ affecting wealth that is realized with time horizon of $n$ periods can be allocated to $n$ consumption risks $\widetilde{x} / n$. This smoothing of the shock plays exactly the same role as a diversification device. The reduction of the risk induces prudent agents to reduce their precautionary savings. In other terms, when risk is realized early in life, consumers are in a position to smooth it over their entire lifetime. On the contrary, when risk is realized during the last period of their life, they will have to absorb each dollar of loss or gain of the lottery into immediate reduction or increase in their consumption. It is intuitive that consumers should care more about such latter risks by saving more.

However, we show that this reasoning holds only when the risk free rate in the economy equals the rate of pure preference for the present. The problem becomes much more complex when this assumption is relaxed. This is easy to understand. When the risk-free rate is just equal to the rate of pure preference for the present, a full early resolution of the uncertainty induces 
consumers to perfectly smooth their consumption over their remaining lifetime. This yields the time diversification presented above. When we relax this assumption, perfect consumption smoothing is not optimal anymore, and the argument cannot be used anymore. We provide a counterexample to this. Finally, we show that the result is robust when we assume for example that the utility function belongs to the HARA class, which includes the logarithmic, the exponential, the quadratic and the power functions.

The course of the paper is the following. In the next section, we introduce the model. Section 3 is devoted to the benchmark case where the risk free rate of the economy equals the rate of pure preference for the present. We show that positive prudence is necessary and sufficient for an early resolution of uncertainty to raise initial consumption. We also show that this result does not hold when the risk free rate is not equal to the rate of pure preference for the present. In section 4, we examine the case of a small risk on wealth, whereas the general necessary and sufficient condition is extracted in section 5. We also explain why our results differ from those of Blundell and Stoker (1999). The last section concludes.

\section{The model}

We consider the standard consumption-saving problem with three dates. The felicity function of the consumer is denoted $u($.$) , and is assumed to be in-$ creasing and concave. The discount factor of utility is denoted $\beta$. The agent is endowed with a flow of income $\widetilde{y}_{t}, t=1,2,3$. Only $\widetilde{y}_{3}$ is uncertain. Let 
$R>0$ denote one plus the risk free rate, and $\widetilde{w}=R^{2} y_{1}+R y_{2}+\widetilde{y}_{3}$ is the future value of the flow of incomes. In the absence of any early resolution of uncertainty, the problem of the consumer is written as

$$
c_{1}^{*} \in \arg \max _{c_{1}} u\left(c_{1}\right)+\beta\left\{\max _{c_{2}} u\left(c_{2}\right)+\beta E u\left(\widetilde{w}-R^{2} c_{1}-R c_{2}\right)\right\} .
$$

Assuming that $u$ is differentiable, the first-order conditions to this program yield

$$
u^{\prime}\left(c_{1}^{*}\right)=\beta R u^{\prime}\left(c_{2}^{*}\right)=(\beta R)^{2} E u^{\prime}\left(\widetilde{w}-R^{2} c_{1}^{*}-R c_{2}^{*}\right)
$$

The special case of the cake-eating problem and its applications to nonrenewable resources are obtained when $R=1$ : the stock of the resource is not productive. In this case the problem is to determine the socially efficient rate of extraction when the stock of the resource is uncertain. Since utility is increasing, we have directly incorporated into the program the fact that the cake will be completely consumed within the three periods. We also implicitly assumed that it is never optimal to run the risk of consuming entirely the cake before date 3 . The assumption $u(0)=-\infty$ is sufficient to guarantee that this is the case.

The objective of the paper is to compare the optimal initial consumption with a late resolution of uncertainty, $c_{1}^{*}$, to the optimal initial consumption $c_{1}^{* *}$ when $\widetilde{w}$ is revealed between date $t=1$ and $t=2$. In this case of early resolution of uncertainty, the consumption problem becomes 


$$
c_{1}^{* *} \in \arg \max _{c_{1}} u\left(c_{1}\right)+\beta E\left\{\max _{c_{2}} u\left(c_{2}\right)+\beta u\left(\widetilde{w}-R^{2} c_{1}-R c_{2}\right)\right\} .
$$

We can solve this problem by backward induction. For each net future value $z=w-R^{2} c_{1}$, we obtain $c_{2}(z)$ by solving

$$
u^{\prime}\left(c_{2}^{* *}(z)\right)=\beta R u^{\prime}\left(z-R c_{2}^{* *}(z)\right)
$$

for each $z$. The optimal early consumption is then obtained by solving the following Euler equation:

$$
u^{\prime}\left(c_{1}^{* *}\right)=\beta R E u^{\prime}\left(c_{2}^{* *}\left(\widetilde{w}-R^{2} c_{1}^{* *}\right)\right) .
$$

The intuition suggests that $c_{1}^{* *}$ is larger than $c_{1}^{*}$ : an earlier resolution of uncertainty makes the future less problematic, something that should induce the agent to reduce his precautionary savings. Because problem (3) is concave in $c_{1}$, this is the case if and only if

$$
u^{\prime}\left(c_{1}^{*}\right) \geq \beta R E u^{\prime}\left(c_{2}^{* *}\left(\widetilde{w}-R^{2} c_{1}^{*}\right)\right)
$$

or, equivalently, if and only if

$$
E u^{\prime}\left(c_{2}^{* *}\left(\widetilde{w}-R^{2} c_{1}^{*}\right)\right) \leq u^{\prime}\left(c_{2}^{*}\right)
$$

In short, the expectation of an early resolution of uncertainty increases initial consumption if and only if it reduces the marginal value of future wealth, which is given by the expected marginal utility of future optimal consumption. 


\section{The benchmark case: $\beta R=1$}

This problem is easiest to solve in the special case with $\beta R=1$, i.e., when the rate of pure preference for the present is equal to the risk free rate of the economy. In the cake-eating problem $(R=1)$, this is the case when the social planner allocates the same utility weight to the successive generations $(\beta=1)$.

When $\beta R=1$, we see from condition (4) that with an early resolution of uncertainty, consumption smoothing is optimal for the last two dates, $c_{2}^{* *}(z)=z-R c_{2}^{* *}(z)=z /(R+1)$. Let

$$
\widetilde{z}^{*}=\widetilde{w}-R^{2} c_{1}^{*}
$$

denote the future wealth net of the future value of initial consumption $c_{1}^{*}$. In the case of a late resolution of uncertainty, the date 2 optimal consumption is given by

$$
u^{\prime}\left(c_{2}^{*}\right)=E u^{\prime}\left(\widetilde{z}^{*}-R c_{2}^{*}\right)
$$

Thus, the comparative static condition (7) can be rewritten as

$$
E u^{\prime}\left(\frac{\widetilde{z}^{*}}{R+1}\right) \leq u^{\prime}\left(c_{2}^{*}\right)
$$

Now, observe that

$$
\begin{aligned}
u^{\prime}\left(c_{2}^{*}\right) & =\frac{R}{R+1} u^{\prime}\left(c_{2}^{*}\right)+\frac{1}{R+1} E u^{\prime}\left(\widetilde{z}^{*}-R c_{2}^{*}\right) \\
& =E\left[\frac{R}{R+1} u^{\prime}\left(c_{2}^{*}\right)+\frac{1}{R+1} u^{\prime}\left(\widetilde{z}^{*}-R c_{2}^{*}\right)\right]
\end{aligned}
$$


The first equality is directly derived from the first order condition (9) for $c_{2}^{*}$. The expectation operator at the end is on a weighted sum of marginal utility, which can itself be interpreted as an expected marginal utility conditional to $\widetilde{z}^{*}=z^{*}$. This reinterpretation is a crucial point, as we will see below. Observe that, if $u^{\prime}$ is convex, this conditional expectation satisfies the following Jensen's inequality:

$$
\frac{R}{R+1} u^{\prime}\left(c_{2}^{*}\right)+\frac{1}{R+1} u^{\prime}\left(z^{*}-R c_{2}^{*}\right) \geq u^{\prime}\left(\frac{z^{*}}{1+R}\right)
$$

Taking the expectation with respect to $\widetilde{z}^{*}$ directly implies necessary and sufficient condition (10). This proves the sufficiency part of the following Proposition.

Proposition 1 Suppose that the rate of pure preference for the present is equal to the risk free rate: $\beta R=1$. Then, an earlier resolution of uncertainty raises initial consumption if and only if the consumer is prudent ( $u^{\prime}$ convex).

Sketch of the proof of necessity: Suppose that $u^{\prime}$ is locally concave around $y$. Take $R=1$ and $\widetilde{z}^{*}=2 y+k \widetilde{\varepsilon}$, with $E \widetilde{\varepsilon}=0$. For $k$ small enough, $c_{2}^{*}, \widetilde{z}^{*} /(R+1)$ and $\widetilde{z}^{*}-R c_{2}^{*}$ are in the neighborhood of $y$. Using Jensen's inequality directly yields the comparative statics condition opposite to (10)

This Proposition provides a new definition of the concept of prudence alternative to Kimball (1990) under which an agent is prudent if and only if adding a zero mean risk to his future income reduces his initial consumption. It is well known from Leland (1968) that a necessary and sufficient condition 
for a risk-averse agent to make positive precautionary savings is the convexity of marginal utility. We showed that the same condition is necessary and sufficient for an earlier resolution of uncertainty to yield an increase in initial consumption, when $\beta R=1$. In the following, we establish a simple intuition for this equivalence. The underlying idea is that information reduces risk. It thus reduces the need for precautionary savings, under prudence.

An early resolution of uncertainty allows for a better smoothing of risks over time and thus should increase consumption under prudence. If uncertainty is realized at the intermediary date $t=2$, rather than at the last date, the consumer can "time diversify" the shock over the last two periods. This means splitting every dollar of loss or gain in wealth into a fifty cents loss or gain in consumption at each date. Our interpretation of the concept of time diversification is as follows. ${ }^{1}$ The marginal value of wealth after date $t=1$ is the discounted value of the expected marginal utility of optimal future consumption $\left(\widetilde{c}_{2}, \widetilde{c}_{3}\right)$. It is given by

$$
E u^{\prime}\left(\widetilde{c}_{2}\right)+\beta E u^{\prime}\left(\widetilde{c}_{3}\right)=(1+\beta) E\left[\frac{1}{1+\beta} u^{\prime}\left(\widetilde{c}_{2}\right)+\frac{\beta}{1+\beta} u^{\prime}\left(\widetilde{c}_{3}\right)\right] .
$$

The bracketed term of the right-hand-side of this equality can be interpreted as the expected marginal utility of a random variable which is distributed as $\left(c_{2},(1+\beta)^{-1} ; c_{3}, \beta(1+\beta)^{-1}\right)$. For a given state $\widetilde{z}^{*}=z^{*}$, this random variable is degenerated at $c_{2}^{* *}=c_{3}^{* *}=z^{*} /(1+R)$ with an early resolution of uncertainty. It takes values $c_{2}^{*}$ and $z^{*}-R c_{2}^{*}$ when the information is not

\footnotetext{
${ }^{1}$ For an exposition of the fallacious interpretations of this concept, see Samuelson (1963). More details are provided in Gollier (2002).
} 
revealed before $t=3$. The point is that under $\beta R=1$, the second "lottery" is a mean-preserving spread of the first. This implies that under prudence, the expected - or discounted - marginal utility is increased by this absence of time diversification, state by state. Taking the expectation with respect to the states of nature yields the result.

Of course, an upper bound to the optimal initial consumption with a resolution of the uncertainty in the middle period is the one that would be optimal without any uncertainty. This is because the value function on wealth inherits prudence from the utility function on consumption. ${ }^{2}$

At this stage, it is important to remember that we only considered the particular case where $\beta R=1$. This is a particular case since when $\beta R \neq 1$ perfect consumption smoothing through time is no more optimal and the time diversification effect cannot be used without an adaptation.

\subsection{A counterexample}

Let us consider the following numerical example. First take the utility function

$$
u(c)=\left\{\begin{array}{lll}
-4+3 c-\frac{c^{2}}{2} & \text { if } & c<2 \\
\ln (c-1) & \text { if } & c \geq 2 .
\end{array}\right.
$$

It yields the following marginal utility function:

$$
u^{\prime}(c)= \begin{cases}3-c & \text { if } \quad c<2 \\ (c-1)^{-1} & \text { if } \quad c \geq 2\end{cases}
$$

\footnotetext{
${ }^{2}$ With the resolution of uncertainty at the middle period, the problem is to maximize $u\left(c_{1}\right)+\beta E v\left(\widetilde{w}-R c_{1}\right)$, where $v(z)=\max _{c} u(c)+\beta u(z-R c)$. It is easy to check that $v^{\prime}$ is convex if $u^{\prime}$ is convex. See Carroll and Kimball (1996).
} 
This marginal utility function is decreasing and convex, i.e., the consumer is risk-averse and prudent. Suppose that the future wealth is distributed as $\widetilde{w}=8.329+\widetilde{\varepsilon}$, with $\widetilde{\varepsilon}=(-0.5,1 / 2 ; 0.5,1 / 2)$. Finally, we suppose that $R=1$ and $\beta=4 / 9$. Thus, we cannot use Proposition 1 to determine the effect of an early resolution of uncertainty on the initial consumption. We solve this problem numerically. In the economy with a late resolution of uncertainty, the optimal stochastic consumption path is as follows:

$$
c_{1}^{*}=4.351-c_{2}^{*}=2.489 \stackrel{c_{31}^{*}=1.989}{c_{32}^{*}=0.989}
$$

In the case of an early resolution of uncertainty, the optimal stochastic consumption path is written as

$$
\begin{array}{ll}
c_{1}^{* *}=4.329 & c_{21}^{* *}=2.771-c_{31}^{* *}=1.729 \\
& c_{22}^{* *}=2.271-c_{32}^{* *}=1.229
\end{array}
$$

We see that $c_{1}^{* *}$ is smaller than $c_{1}^{*}$, in spite of positive prudence.

\section{The case of small risks when $\beta R \neq 1$}

The previous example has demonstrated that prudence is not sufficient to sign the effect of an early resolution of uncertainty for any probability distribution when $\beta R \neq 1$. In this section, we derive the necessary and sufficient condition that permits to sign this effect when $\beta R$ is arbitrary, but $\widetilde{w}$ is a small risk. 
It will be convenient to define $\widetilde{\varepsilon}, k$ and $z^{*}$ so that

$$
\widetilde{z}^{*}(k)=z^{*}+k \widetilde{\varepsilon} \text { with } E \widetilde{\varepsilon}=0 .
$$

The marginal value of wealth before date $t=2$ with an early resolution of uncertainty is denoted

$$
j^{* *}(k) \equiv E u^{\prime}\left(c_{2}^{* *}\left(\widetilde{z}^{*}(k)\right)\right),
$$

where function $c_{2}^{* *}(z)$ is defined by (4). With a late resolution of uncertainty, the marginal value of wealth before $t=2$ equals

$$
j^{*}(k)=u^{\prime}\left(c_{2}^{*}(k)\right)=\beta R E u^{\prime}\left(\widetilde{z}^{*}(k)-R c_{2}^{*}(k)\right),
$$

where $c_{2}^{*}(k)$ is the optimal consumption at $t=2$ when the net wealth at $t=3$ is distributed as $\widetilde{z}^{*}(k)$.

The early resolution of uncertainty raises initial consumption when the future risk is small if and only if $j^{* *}(k)$ is smaller than $j^{*}(k)$ in the neighborhood of $k=0$. It is easy to check that

$$
j^{* *}(0)=j^{*}(0)=u^{\prime}\left(c_{20}\right)
$$

with $c_{20}=c_{2}^{* *}\left(z^{*}\right)=c_{2}^{*}(0)$. Turning to the first derivatives, we obtain

$$
\left.\frac{\partial j^{* *}}{\partial k}\right|_{k=0}=\left.\frac{\partial j^{*}}{\partial k}\right|_{k=0}=0
$$

Thus, we are forced to examine the second-order effect of risk. After tedious manipulations, we obtain the following Proposition. It relies on the indexes 
of absolute risk aversion and absolute prudence which are defined as

$$
A(c)=\frac{-u^{\prime \prime}(c)}{u^{\prime}(c)} \text { and } P(c)=\frac{u^{\prime \prime \prime}(c)}{-u^{\prime \prime}(c)} \text {. }
$$

We hereafter assume that these functions exists, i.e., that $u$ is thrice differentiable.

Proposition 2 Suppose that the risk on future wealth is small. Then, an early resolution of uncertainty raises initial consumption if and only if

$$
P\left(c_{2}\right)-R P\left(c_{3}\right) \leq 2 \frac{A\left(c_{2}\right)}{A\left(c_{3}\right)} P\left(c_{3}\right)
$$

for any pair $\left(c_{2}, c_{3}\right)$ that satisfies the first-order condition $u^{\prime}\left(c_{2}\right)=\beta R u^{\prime}\left(c_{3}\right)$.

Proof: Take $c_{2}=c_{20}$ and so $c_{3}=z^{*}-R c_{2}$. Fully differentiating condition (19) twice around $k=0$ yields

$$
\left.\frac{\partial^{2} j^{* *}}{\partial k^{2}}\right|_{k=0}=\sigma^{2} \beta R \frac{u^{\prime \prime}\left(c_{2}\right) u^{\prime \prime \prime}\left(c_{3}\right)\left[A\left(c_{2}\right)\right]^{2}+\beta R u^{\prime \prime}\left(c_{3}\right) u^{\prime \prime \prime}\left(c_{2}\right)\left[A\left(c_{3}\right)\right]^{2}}{\left[A\left(c_{2}\right)+R A\left(c_{3}\right)\right]^{2}\left[u^{\prime \prime}\left(c_{2}\right)+\beta R^{2} u^{\prime \prime}\left(c_{3}\right)\right]},
$$

where $\sigma^{2}$ is the variance of $\widetilde{\varepsilon}$. The equivalent manipulation on $j^{*}$ yields

$$
\left.\frac{\partial^{2} j^{*}}{\partial k^{2}}\right|_{k=0}=\sigma^{2} \frac{u^{\prime \prime}\left(c_{2}\right) u^{\prime \prime \prime}\left(c_{3}\right) A\left(c_{3}\right)}{\left[A\left(c_{2}\right)+R A\left(c_{3}\right)\right] u^{\prime \prime}\left(c_{3}\right)}
$$

Using first-order condition $\beta R=u^{\prime}\left(c_{2}\right) / u^{\prime}\left(c_{3}\right)$, it is easy to check that the right-hand-side of (25) is smaller than the right-hand-side of (26) if and only if condition (24) is satisfied.

When $\beta R=1$, condition $(24)$ becomes $(1-R) P\left(c_{2}\right) \leq 2 P\left(c_{2}\right)$, since $\beta R=1$ implies that $c_{2}=c_{3}$. Because $R>-1$, this condition is equivalent 
to nonnegative prudence. But this is true only when $\beta R=1$. For example, utility function (14) does not satisfy this condition when $\beta R \neq 1$ in spite of nonnegative prudence. Indeed, for $\beta R<1$, as in the counterexample, the rate of pure preference for the present is larger than the interest rate, which implies that the relevant domain of $\left(c_{2}, c_{3}\right)$ is such that $c_{2} \geq c_{3}$. Now, observe that, for utility function (14), prudence is zero for small $c_{3}$. Thus, the righthand side of inequality (24) is zero in this region, whereas the left-hand-side is positive, thereby violating the condition.

We can extract from this an intuition for why prudence is not sufficient when $\beta R$ is different from unity. The early resolution of uncertainty allows for transferring part of the risk to the second period. This is a timediversification device, which tends to reduce the precautionary saving under positive prudence. But when $\beta R \neq 1$, it transfers risk to different consumption levels. If it happens that the degree of prudence is much larger at date 2 than at date $3\left(P\left(c_{2}\right) \ggg P\left(c_{3}\right)\right)$, this risk transfer may generate an increase in the marginal value of wealth, which is measured by the expected marginal utility of future consumption. This is exactly the way by which we built the counterexample. Observe that the early resolution of uncertainty transfers half of the risk from a region where prudence is zero $\left(c_{3}<2\right)$ to a region where prudence is positive $\left(c_{2}>2\right)$. Condition $(24)$ thus puts an upper bound to the degree of prudence in the region where part of the risk is transferred thanks to the early resolution of uncertainty. Note also that it is necessary that the utility function exhibits increasing absolute prudence to 
generate such a counterexample.

\section{The general case within $\beta R \neq 1$}

The general problem that we have to solve takes the following form. We want to guarantee that, for any distribution of the net wealth $\widetilde{z}$, and for any $c_{2}$, the following condition is satisfied:

$$
\beta R E u^{\prime}\left(\widetilde{z}-R c_{2}\right)-u^{\prime}\left(c_{2}\right)=0 \Rightarrow E u^{\prime}\left(c_{2}^{* *}(\widetilde{z})\right)-u^{\prime}\left(c_{2}\right) \leq 0
$$

where function $c_{2}^{* *}($.$) is defined in (4). The equality to the left of this con-$ dition states that $c_{2}=c_{2}^{*}$ is the optimal consumption at date 2 without information. The inequality to the right states that the marginal value of wealth is smaller with information than without information.

Consider a specific $c_{2}$. Using the hyperplane separation theorem, as in Pratt and Zeckhauser (1986) and Gollier, Jullien and Treich (2000), this condition holds for any $\widetilde{z}$ if and only if there exists a scalar $\lambda=\lambda\left(c_{2}\right)$ such that

$$
G\left(z, c_{2}, \lambda\right)=u^{\prime}\left(c_{2}^{* *}(z)\right)-u^{\prime}\left(c_{2}\right)-\lambda\left[\beta R u^{\prime}\left(z-R c_{2}\right)-u^{\prime}\left(c_{2}\right)\right] \leq 0
$$

for all $z$. Define $\widehat{z}$ such that $\beta R u^{\prime}\left(\widehat{z}-R c_{2}\right)=u^{\prime}\left(c_{2}\right)$. We can interpret $\widehat{z}$ as the precautionary equivalent wealth to $\widetilde{z}$. Observe that, by definition, $c_{2}^{* *}(\widehat{z})=c_{2}$. This implies that $G\left(\widehat{z}, c_{2}, \lambda\right)=0$. Therefore, in order to guarantee that $G$ is nonpositive in the neighborhood of $z=\widehat{z}$, we need that

$$
0=\frac{\partial G}{\partial z}\left(\widehat{z}, c_{2}, \lambda\right)=u^{\prime \prime}\left(c_{2}\right) c_{2}^{* *^{\prime}}(\widehat{z})-\lambda \beta R u^{\prime \prime}\left(\widehat{z}-R c_{2}\right)
$$


This allows us to extract the only possible candidate for $\lambda$ that could satisfy condition (28). Using condition $\beta R u^{\prime}\left(\widehat{z}-R c_{2}\right)=u^{\prime}\left(c_{2}\right)$, we get

$$
\lambda=\frac{A\left(c_{2}\right)}{A\left(\widehat{z}-R c_{2}\right)} c_{2}^{* *^{\prime}}(\widehat{z})=c_{3}^{* *^{\prime}}(\widehat{z}),
$$

where $c_{3}^{* *}(z)=z-R c_{2}^{* *}(z)$. Indeed, fully differentiating condition (4) yields

$$
c_{2}^{* * \prime}(z)=\frac{A\left(z-R c_{2}^{* *}(z)\right)}{A\left(c_{2}^{* *}(z)\right)+R A\left(c_{3}^{* *}(z)\right)} \text { and } c_{3}^{* * \prime}(z)=\frac{A\left(c_{2}^{* *}(z)\right)}{A\left(c_{2}^{* *}(z)\right)+R A\left(c_{3}^{* *}(z)\right)} .
$$

Notice that conditions (31) determine the optimal allocation of risk $\widetilde{z}$ over dates $t=2$ and 3. As suggested by the intuition, it is optimal to allocate a larger share of the risk at time where risk aversion is lower. Combining these observations allows us to write the following Lemma.

Lemma 1 Consider a given pair $(\beta, R)$ and a twice differentiable, increasing and concave utility function $u$. An early resolution of uncertainty raises initial consumption for any distribution of net wealth $\widetilde{z}$ if and only if for all $z, \widehat{z}$, we have

$$
u^{\prime}\left(c_{2}^{* *}(z)\right) \leq\left(1-c_{3}^{* *^{\prime}}(\widehat{z})\right) u^{\prime}\left(c_{2}^{* *}(\widehat{z})\right)+c_{3}^{* *^{\prime}}(\widehat{z}) \beta R u^{\prime}\left(z-R c_{2}^{* *}(\widehat{z})\right)
$$

where $c_{2}^{* *}($.$) is defined in (4) and c_{3}^{* *^{\prime}}($.$) is defined by condition (31).$

Notice first that the sufficiency of (32) is immediately obtained by taking its expectation with respect to $z=\widetilde{z}$, and with a constant $\widehat{z}$ such that $c_{2}^{* *}(\widehat{z})=c_{2}^{*}$. It yields

$$
\begin{aligned}
E u^{\prime}\left(c_{2}^{* *}(\widetilde{z})\right) & \leq\left(1-c_{3}^{* *^{\prime}}(\widehat{z})\right) u^{\prime}\left(c_{2}^{*}\right)+c_{3}^{* *^{\prime}}(\widehat{z}) \beta R E u^{\prime}\left(\widetilde{z}-R c_{2}^{*}\right) \\
& =u^{\prime}\left(c_{2}^{*}\right),
\end{aligned}
$$


where we used the first-order condition (2) for $c_{2}^{*}$. This shows that the early resolution of uncertainty reduces the marginal value of wealth if condition (32) is satisfied. The difficult part of the proof above was to show that this condition is also necessary.

Keep in mind that we can interpret $c_{2}^{* *}(\widehat{z})$ as $c_{2}^{*}$, the optimal consumption at $t=2$ without information. The necessary and sufficient condition states that, in each state of nature $z$, the marginal utility of optimal consumption with information must be less than a weighted average of discounted marginal utilities at date $t=2$ and 3 without information. The discount rate is $\beta R$, and the implicit probabilities are $1-c_{3}^{* * \prime}(\widehat{z})$ and $c_{3}^{* * \prime}(\widehat{z})$. Again, it is easy to get back the case $\beta R=1$, since it implies $c_{2}^{* *}(z)=c_{3}^{* *}(z)=z /(1+R)$ and $c_{3}^{* *^{\prime}}=1 /(1+R)$. In such a case, condition (32) is formally equivalent to condition (12), which holds if and only if $u^{\prime}$ is convex.

Our necessary and sufficient condition is still complex, as it requires that a bivariate function $F(.,$.$) be nonpositive, with$

$$
F(z, \widehat{z})=u^{\prime}\left(c_{2}^{* *}(z)\right)-\left(1-c_{3}^{* *^{\prime}}(\widehat{z})\right) u^{\prime}\left(c_{2}^{* *}(\widehat{z})\right)-c_{3}^{* *^{\prime}}(\widehat{z}) \beta R u^{\prime}\left(z-R c_{2}^{* *}(\widehat{z})\right)
$$

By construction, we have that $F(\widehat{z}, \widehat{z})=F_{1}(\widehat{z}, \widehat{z})=0$. Thus, a necessary condition is that $F_{11}(\widehat{z}, \widehat{z})$ be nonpositive. After some manipulations, it can be checked that this necessary condition is nothing else than condition (24), the necessary and sufficient condition for small risk on net wealth.

Because of the complexity of the necessary and sufficient condition (32), we now derive a simpler sufficient condition from it. The necessary and suffi- 
cient condition requires that $F(z, \widehat{z})$ be uniformly nonpositive. Our sufficient condition requires that $F_{1}(z, \widehat{z})$ has the same sign as $\widehat{z}-z$, which is stronger than necessary. Replacing $u^{\prime}\left(c_{2}^{* *}(z)\right)$ by $\beta R u^{\prime}\left(c_{3}^{* *}(z)\right)$ in (34), we have

$$
F_{1}(z, \widehat{z})=\beta R\left[u^{\prime \prime}\left(z-R c_{2}^{* *}(z)\right) c_{3}^{* * \prime}(z)-u^{\prime \prime}\left(z-R c_{2}^{* *}(\widehat{z})\right) c_{3}^{* *^{\prime}}(\widehat{z})\right]
$$

Suppose that the utility function belongs to the class of HARA utility functions, with $u^{\prime}(c)=(\eta+c / \gamma)^{-\gamma}$. This family of functions gathers all familiar utility functions as exponential, quadratic, power and logarithmic ones. As it is well-known since Wilson (1968), all efficient risk-sharing rules are linear when the utility function belongs to the HARA class. This implies that $c_{3}^{* *^{\prime}}(z)=c_{3}^{* *^{\prime}}(\widehat{z})$ and yields

$$
F_{1}(z, \widehat{z})=\beta R\left[u^{\prime \prime}\left(z-R c_{2}^{* *}(z)\right)-u^{\prime \prime}\left(z-R c_{2}^{* *}(\widehat{z})\right)\right] c_{3}^{* *^{\prime}}(z)
$$

If $z$ is larger than $\widehat{z}, c_{2}^{* *}(z)$ is larger than $c_{2}^{* *}(\widehat{z})$ and

$$
u^{\prime \prime}\left(z-R c_{2}^{* *}(z)\right)-u^{\prime \prime}\left(z-R c_{2}^{* *}(\widehat{z})\right) \leq 0
$$

for the subset of HARA functions that exhibit prudence, as is the case for exponential, power and logarithmic functions. Therefore, $F_{1}(z, \widehat{z})$ is negative when $z>\widehat{z}$. Symmetrically, $F_{1}(z, \widehat{z})$ is positive when $z<\widehat{z}$. Because $F(\widehat{z}, \widehat{z})=0$, this is sufficient for $F$ to be uniformly nonpositive. Proposition 1 implies that an early resolution of uncertainty raises initial consumption. From these computations, it is easy to check that prudence is also necessary when the utility function is restricted to be in the HARA class. 
Proposition 3 Suppose that $u$ is HARA. Then, an earlier resolution of uncertainty raises initial consumption if and only if the consumer is prudent.

Notice that we don't need to have any information about the values of $\beta$ and $R$ to conclude the above statement in the HARA case. In spite of the common use of the HARA assumption in macroeconomics and in finance, the empirical tests of this assumption are scarce in the literature. Guiso and Paiella (2000) rejects it by using panel data of Italian households. But this assumption is sustained by the casual observation that the level of the risk free rate and the equity premium do not show any decreasing trend over the past century in spite of the important growth of GDP per capita experienced during the period. ${ }^{3}$

Recently, Blundell and Stoker (1999) considered the same three-period framework to provide an approximate solution for optimal consumption choice for preferences that display constant relative risk aversion, which is a special case of HARA. They used this framework to derive the relation between information and consumption. They gave numerical examples where risk resolving earlier in one's life leads to a lower initial consumption. Their numerical results are thus a priori contradictory with Proposition 3. We now explain why.

Suppose, as in Blundell-Stoker, that total income is made of three parts $y_{1}, \widetilde{y}_{2}$ and $\widetilde{y}_{3}$, where income $\widetilde{y}_{t}$ is received in period $t$. The total future wealth

\footnotetext{
${ }^{3}$ In Eeckhoudt, Gollier and Treich (2001), we easily derive from Lemma 1 that prudence together with either the concavity or the convexity of absolute risk tolerance is sufficient to sign the comparative statics analysis.
} 
is thus

$$
\widetilde{w}=R^{2} y_{1}+R \widetilde{y}_{2}+\widetilde{y}_{3}
$$

To examine the effect of the resolution of uncertainty, Blundell and Stoker compare scenario $1\left(\widetilde{y}_{2}=y+\widetilde{\varepsilon}, \widetilde{y}_{3}=y\right)$ to scenario $2\left(\widetilde{y}_{2}=y, \widetilde{y}_{3}=y+\widetilde{\varepsilon}\right)$, with $E \widetilde{\varepsilon}=0$. Obviously, the uncertainty is revealed earlier in scenario 1 . But there is a second effect in the Blundell-Stoker model. The point is that the ex ante distribution of total future wealth is not the same in the two scenarios. By transferring the risk from period 3 to period 2, there is also an increase in risk on wealth by a factor $R>1$. In order to isolate the effect of an early information, it is crucial to let the prior distribution of aggregate wealth unchanged. The effect exhibited by the numerical simulations of Blundell-Stoker is in fact a precautionary effect: transferring uncertain incomes from date 3 to date 2 increases the aggregate future risk, which induces prudent agents to raise their precautionary saving. Our work has shown that Blundell and Stoker would have obtained the opposite result if they would have selected a sufficiently smaller risk free rate.

\section{Conclusion}

Because labour income risks cannot be insured, workers must accumulate precautionary savings. But risks that are resolved early in their life cycle can be self-insured through time diversification. Time diversification is the mechanism by which large risks on wealth are disseminated into small variations 
in consumption over the entire lifetime from the date at which these risks are realized. It is a substitute to market insurance. As a consequence, the ability of workers to transfer uninsurable risks through time by an efficient contingent saving/borrowing strategy should reduce their precautionary savings ex ante. Because this mechanism works better when the resolution of the uncertainty arises earlier in life, any earlier resolution of uncertainty should induce consumers to reduce their initial savings. We showed in this paper that this intuition is sustained by the theory at least in two special cases. An early resolution of uncertainty reduces the initial saving of prudent consumers if either their utility function exhibits HARA, or if the rate of pure preference for the present equals the risk free rate.

We considered the simplest model that allowed us to examine the relationship between the timing of the resolution of uncertainty and savings. Of course, it is too simple to examine more empirical questions. Interested readers should rely on the existing literature in which the classical life cycle model is calibrated with various stochastic income processes. Several extensions of this theoretical work can be considered. For example, we limited the analysis to the perfect resolution of uncertainty, i.e., the uncertainty is fully eliminated either at date 1 or at date 2. In Eeckhoudt, Gollier and Treich (2001), we show that our results are not robust to the introduction of a partial resolution of uncertainty. One should also introduce a liquidity constraint for the sake of realism. Finally, one could reinterpret our model as the description of a consumption-saving problem in which the consumer does not know the 
shape of their future preferences. In our additive model, the date- 2 utility function takes the form $v(., y)=u(.+y)$, where $y$ is some parameter whose value would be unknown at date 0 . A possible extension of this work would be to consider more general functional forms for $v$, as considered for example by Ando, Guiso and Terlizzese (1994). 


\section{References}

Ando, A., L. Guiso and D. Terlizzese, 1994, Young household's saving and the life cycle of opportunities, in Ando et al. (Eds), Saving and the accumulation of wealth (Cambridge University Press).

Blundell, R. and T. M. Stoker, 1999, Consumption and the timing of income risk, European Economic Review 43, 475-507.

Campbell, J. and N.G. Mankiw, 1990, Permanent income, current income, and consumption, Journal of Business and Economic Statistics 8, 265-278.

Carroll, C.D., 1994, How does future income affect consumption? Quarterly Journal of Economics 109, 111-48.

Carroll, C.D., 1997, Buffer-stock saving and the life cycle/permanent income hypothesis, Quarterly Journal of Economics 112, 1-55.

Carroll, C.D. and M. Kimball, 1996, On the concavity of the consumption function, Econometrica 64, 981-92.

Deaton, A.S., 1992, Understanding consumption (Oxford University Press, Oxford).

Drèze, J.H. and F. Modigliani, 1972, Consumption decisions under uncertainty, Journal of Economic Theory 5, 308-335.

Eeckhoudt, L., C. Gollier and N. Treich, 2001, Optimal consump- 
tion and the timing of the resolution of uncertainty, mimeo. Downloadable at http://w3.toulouse.inra.fr/leerna/

Epstein, L.G., 1980, Decision making and the temporal resolution of uncertainty, International Economic Review 21, 269-83.

Gollier, C., 2002, Time diversification, liquidity constraints, and decreasing aversion to risk on wealth, Journal of Monetary Economics, forthcoming.

Gollier, C., Jullien, B. and N. Treich, 2000, Scientific progress and irreversibility: An economic interpretation of the 'Precautionary Principle', Journal of Public Economics 75, 229-53.

Guiso, L. and M. Paiella, 2000, Risk aversion, wealth and financial market imperfections, mimeo, University of Sassari, Italy.

Kimball, M., 1990, Precautionary savings in the small and in the large, Econometrica 58, 53-73.

Kimball, M.S., 1993, Standard risk aversion, Econometrica 61, $589-611$.

Leland, H., 1968, Savings and uncertainty: The precautionary demand for savings, Quarterly Journal of Economics 45, 62136.

Pratt, J.W. and R. Zeckhauser, 1987, Proper risk aversion, Econometrica, 143-54. 
Rothschild, M. and J. Stiglitz, 1970, Increasing risk: I. A definition, Journal of Economic Theory 2, 225-243.

Samuelson, P.A., 1963, Risk and uncertainty: The fallacy of the law of large numbers, Scientia 98, 108-113.

Skinner, J., 1988, Risky income, life cycle consumption, and precautionary savings, Journal of Monetary Economics 22, 23798.

Wilson, R., 1968, The theory of syndicates, Econometrica 36, 113-132. 